\title{
Spin-dependent photoconductivity in CVD- and MBE-grown silicon-on-sapphire
}

\author{
C O'Raifeartaigh $\dagger$, L Bradley $\dagger$, R C Barklie $\nmid$, A M Hodge $\nmid$ and \\ E D Richmond $\S$
}

\author{
† Physics Department, Trinity College, Dublin 2, Ireland \\ Defence Research Agency, Malvern, UK \\ $\S$ Naval Research Laboratory, Code 6816, Washington, DC 20375-5000, USA
}

Received 16 December 1994, in final form and accepted for publication 26 July 1995

\begin{abstract}
Spin-dependent photoconductivity is observed in (100) silicon films grown on sapphire by CVD and MBE. The CVD films are either in their as-grown state or have undergone single or double solid-phase epitaxial regrowth. For all samples a resonant decrease in photoconductivity is observed at a field of about $0.34 \mathrm{~T}$ for a microwave frequency of about $9.6 \mathrm{GHz}$ and at about $3.3 \mathrm{mT}$ when the frequency is about $92 \mathrm{MHz}$. The fractional change in photoconductivity at resonance is measured as a function of the magnetic field strength, microwave or radiofrequency power, temperature, light intensity and sample voltage. The results are interpreted in terms of a quantum mechanical treatment of the pair model of Kaplan, Solomon and Mott and values are extracted for the spin relaxation time, pair dissociation rate and singlet recombination rate. In some samples a resonant change in dark conductivity is also observed and interpreted.
\end{abstract}

\section{Introduction}

Electron paramagnetic resonance (EPR) is a powerful technique for obtaining information about the nature of paramagnetic defects and in view of the interest in identifying and removing the defects in the silicon film of silicon-on-sapphire (SOS) it is surprising that there is, to our knowledge, no published paper on EPR measurements of defects in SOS. The reason for this is probably either that there are too few paramagnetic defects present in the Si film for any signal to be seen or that strong signals from impurities in the sapphire completely obscure the much weaker ones from the defects in the film. There is, however, a magnetic resonance technique called electrically detected EPR (EDEPR) which not only is more sensitive than EPR but is also more selective as it detects only those defects which affect the electrical conductivity of the sample. The essence of this technique, one of the first reports of which is by Lepine [1], is to detect the resonant change in this conductivity which occurs when magnetic resonance is induced in the usual way. In nearly all cases this resonant change is associated with a resonant change in the recombination rate of excess electrons and holes which must be optically or, in the case of rectifying junctions, electrically injected. In such cases the effect is also known as spin-dependent recombination (SDR) and when optical injection is involved, as spin-dependent photoconductivity (SDPC).
The first aim of this work is to use the technique of EDEPR to identify some of the recombination centres in the siilicon film or at its interfaces for a variety of SOS sampies. These samples either had the Si film grown by chemical vapour deposition (CVD) and were in their as-grown state or had undergone solid-phase epitaxial regrowth (SPEG), double solid-phase epitaxial regrowth (DSPEG) or were grown by molecular beam epitaxy (MBE) to three different thicknesses. We wished to determine whether or not these centres are the same in all samples and to see the effect of changing the growth process.

The second aim is to investigate the mechanism of the EDEPR. In the few cases where the necessary measurements have been made [2-6] it has been found that the magnitude of the EDEPR signal is approximately independent of the magnitude of the magnetic field at which the resonance occurs. This was first explained by the model (KSM) proposed by Kapian et al [2], in which recombination of excess carriers takes place via an intermediate pair state having an electron and hole close together. L'vov et al [4] developed a quantum mechanical treatment of the KSM model which also takes account of spin relaxation. More recentiy Rong et al [7] proposed that in $\mathrm{Si} \mathrm{p}-\mathrm{n}$ diodes at room temperature the pair consists of an electron (or hole) trapped in a shallow excited state of a recombination centre. We wish to test these models by investigating the dependence of the resonance signal on the 
Table 1. The labels and characteristics of the SOS wafers.

\begin{tabular}{|c|c|c|c|c|}
\hline Label & $\begin{array}{l}\text { Growth } \\
\text { method }\end{array}$ & $\begin{array}{l}\text { Extra } \\
\text { treatment }\end{array}$ & $\begin{array}{l}\text { Si film } \\
\text { thickness } \\
(\mu \mathrm{m})\end{array}$ & $\begin{array}{l}\text { Conductivity } \\
\left(\Omega \mathrm{cm}^{-1}\right)\end{array}$ \\
\hline $\left.\begin{array}{l}A 1 \\
A 2 \\
S 1 \\
\text { S2 } \\
D 1 \\
D 2 \\
D 3\end{array}\right\}$ & CVD & $\begin{array}{l}\text { None } \\
\text { None } \\
\text { SPEG } \\
\text { SPEG } \\
\text { DSPEG } \\
\text { DSPEG } \\
\text { DSPEG }\end{array}$ & 0.285 & $\begin{array}{l}0.03 \\
3700 \\
1600 \\
1800 \\
0.04 \\
0.03 \\
1700\end{array}$ \\
\hline $\left.\begin{array}{l}M 1 \\
M 2 \\
M 3\end{array}\right\}$ & MBE & $\begin{array}{l}\text { None } \\
\text { None } \\
\text { None }\end{array}$ & $\begin{array}{l}0.5 \\
0.3 \\
0.15\end{array}$ & $\begin{array}{l}0.1 \\
0.03 \\
0.003\end{array}$ \\
\hline
\end{tabular}

magnetic field at resonance, microwave or radiofrequency power, temperature, light intensity and sample voltage.

\section{Sample preparation, structure and $I-V$ characteristics}

Table 1 lists the wafers studied in this work. In several cases more than one sample from a given wafer was studied but as the results for such samples are similar we just label them with the symbol of the wafer from which they came. All wafers consist of (100) silicon on (1012) sapphire. The CVD SOS $3^{\prime \prime}$ wafers were obtained from Union Carbide in their as-grown state. S1, S2, D1, D2 and D3 were then implanted at room temperature with $8 \times 10^{14} \mathrm{~cm}^{-2}$ of $185 \mathrm{keV} \mathrm{Si}^{+}$ions to amorphize all but a thin surface region of the silicon film. The film was then epitaxially regrown by annealing the wafer in nitrogen at $900^{\circ} \mathrm{C}$ for $20 \mathrm{~s}$ to complete the SPEG process for $\mathrm{S} 1$ and $\$ 2$. The samples DI, D2 and D3 undergoing the DSPEG process were given a further implant at room temperature of $2 \times 10^{15} \mathrm{~cm}^{-2}$ of 100 $\mathrm{keV} \mathrm{Si}^{+}$ions in order to amorphize the surface layer which was then regrown by annealing the wafer under the same conditions as those above. Contact regions, $3 \mathrm{~mm} \times 3 \mathrm{~mm}$ square, were then formed by implanting these areas with $2 \times 10^{15} \mathrm{~cm}^{-2}$ of $30 \mathrm{keV} \mathrm{B}^{+}$and annealing in argon at $900^{\circ} \mathrm{C}$ for $30 \mathrm{~min}$ to form heavily doped $\mathrm{p}$-type regions onto which was deposited an aluminium film. Samples of dimensions $9 \mathrm{~mm} \times 3 \mathrm{~mm}$, with a contact pad at each end, were cut from the wafer. These processing steps were carried out at the Defence Research Agency, Malvern.

The wafers M1, M2 and M3 were prepared at the Naval Research Laboratory, Washington, by growing the silicon film by MBE. Each sapphire substrate was annealed at $1415-1450^{\circ} \mathrm{C}$ in ultrahigh vacuum before the silicon was deposited on the substrate at $750^{\circ} \mathrm{C}$. Further details are given elsewhere $[8,9]$. Samples, typically $8 \mathrm{~mm} \times 5 \mathrm{~mm}$ in size, were cleaved from these wafers. Two small aluminium pads were evaporated onto each sample which was then annealed in nitrogen for $10 \mathrm{~min}$ at $450^{\circ} \mathrm{C}$. To measure the current through the silicon layer leads were attached with silver paint to each aluminium pad on both the CVD- and MBE-grown samples.

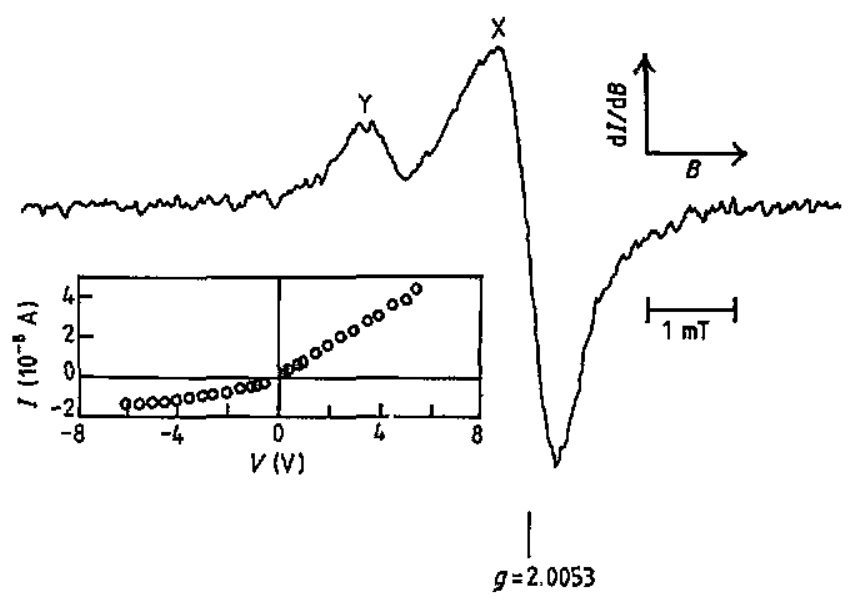

Figure 1. Resonant change in dark current at constant voltage, $V=-4 V$, for sample $A 1$. No signal was detected for positive $V . v \simeq 9.6 \mathrm{GHz}, B \|[011]$. The insert shows the $1-V$ characteristic for this sample.

Table 1 gives the average conductivity in the darkhereafter just referred to as the conductivity-which was measured for each type of sample, except M1 and M3, by a four-point probe. For Ml and M3 the conductivity was determined from the dark current, $I$, voltage, $V$, characteristic. For all samples, except for those from the low-conductivity $\mathrm{CVD}$ wafers $\mathrm{Al}, \mathrm{D} 1$ and $\mathrm{D} 2$, the $I-V$ characteristic is linear. However, the current through $A 1$, D1 and D2 often tends to saturate as $V$ is increased for one polarity of $V$; an example of this behaviour is shown in the inset of figure 1. The most likely explanation for the saturation is that one of the contacts behaves like a reverse-biased diode. In some of these samples the current tends to saturate for both polarities of $V$ which indicates that both contacts then behave like diodes. However, in all samples the photocurrent, $I_{\mathrm{p}}$, varies linearly with voltage for both polarities, at least at the light intensities of 50$80 \mathrm{~mW} \mathrm{~cm}^{-2}$ mostly used in the later experiments. At these light levels the photocurrent considerably exceeds the dark current, $I$, in the samples $\mathrm{Al}, \mathrm{D} 1$ and $\mathrm{D} 2$, is greater than or approximately equal to $I$ in M1, M2 and M3 and is of the order of $1 \%$ of $I$ in the high-conductivity wafers A2, S1, S2 and D3.

The structural characteristics of equivalent as-grown CVD films and the effects of SPEG and DSPEG have been reported elsewhere [10] as have the structural features of equivalent $\mathrm{MBE}$-grown samples $[8,9,11-13]$, so here we just give a brief summary of those features of relevance to the present study. SPEG is found to greatly reduce the microtwin density close to the silicon/sapphire interface, but twins remaining in the top crystalline layer propagate through the film during regrowth. The layers regrown after DSPEG are relatively free of twins but contain dislocation arrays emanating from the sapphire interface.

The microtwin differential volume fraction, which represents the percentage of material which is twinned, in MBE SOS falls off more rapidly with distance from the silicon/sapphire interface than it does in as-grown CVD SOS and at any point gets progressively smaller on going from CVD SOS $(0.55 \mu \mathrm{m})$ to MBE SOS $(0.15 \mu \mathrm{m})$ to MBE SOS $(0.55 \mu \mathrm{m})$. The fact that microtwins are less prevalent 
in MBE SOS than in CVD SOS has led to the conclusion that strain relief by dislocation formation is more important in the former than in the latter $[9,11]$.

\section{Experimental techniques}

The conductivity of the silicon was monitored by using a current amplifier to measure, at constant voltage, the current through the silicon film. Excess carriers were created with light, from a $360 \bar{W}$ tungsten filament lamp, which was filtered by a copper sulphate solution to remove the longwavelength output. A resonance was detected at a magnetic field, $B$, of about $0.34 \mathrm{~T}$ when irradiating the sample with microwaves of frequency $\simeq 9.6 \mathrm{GHz}$ in a $\mathrm{TE}_{011}$ mode cavity and also at $B \simeq 3.3 \mathrm{mT}$ using a radiofrequency oscillator of $92 \mathrm{MHz}$ in series with a $10 \mathrm{~W}$ power amplifier to drive a tuned coil. Field modulation at about $1 \mathrm{kHz}$ was normally used together with lock-in detection, but a PIN diode was occasionally used instead to modulate the microwave power. The $g$ value $\left(=h v / \mu_{\mathrm{B}} \bar{B}\right)$, which is found from the microwave frequency $\nu$ and line position $B$, and the linewidth were determined from the spectrum once the magnetic field scale had been calibrated using a proton NMR probe. The field homogeneity over the sample is better than 1 in $3 \times 10^{4}$ as is usual for magnetic resonance experiments. These measurements were made at Trinity. College, Dublin.

\section{Results}

\subsection{Resonant change in the conductivity}

Figure 1 shows the resonant change in the dark. current, at constant voltage, in a sample from wafer $\mathrm{A} 1$; the microwave frequency was about $9.6 \mathrm{GHz}$ and field modulation at $1 \mathrm{kHz}$ was used; here and elsewhere, unless otherwise stated, the measurements were made at room temperature. This as-received CVD-grown sample has a nonlinear $I-V$ characteristic only when the voltage, $V$, is negative as shown in figure 1, and it was for this polarity only that the signal was observed. Most of the samples from the other two low conductivity, CVD-grown wafers, D1 and D2, exhibited similar nonlinear $I-V$ characteristics and these also gave the same signals in the dark. In some of the A1, D1 or D2 samples the current tended to saturate as the voltage was increased for both polarities of $V$ and in these cases the same type of signal was seen for both polarities. That the signal is associated with the nonlinearity of the $I-V$ characteristic rather than with the dark current as such is reinforced by the fact that no signals were seen in the dark for the other CVD-grown samples whose much higher conductivity meant that, for the same voltages as before, the dark currents were much larger than in A1, D1 or D2. The $I-V$ characteristics of the MBE-grown samples were very nearly linear and any resonant change in the dark current was either very small or unobservable.

Figure 1 shows that the signal, for the magnetic field $B \|[011]$, consists of two features X and Y. For this field direction $X$ is a slightly asymmetric line with peak-topeak width, $\Delta B_{\mathrm{pp}}=0.66 \mathrm{mT}$, and a zero crossing $\mathrm{g}$

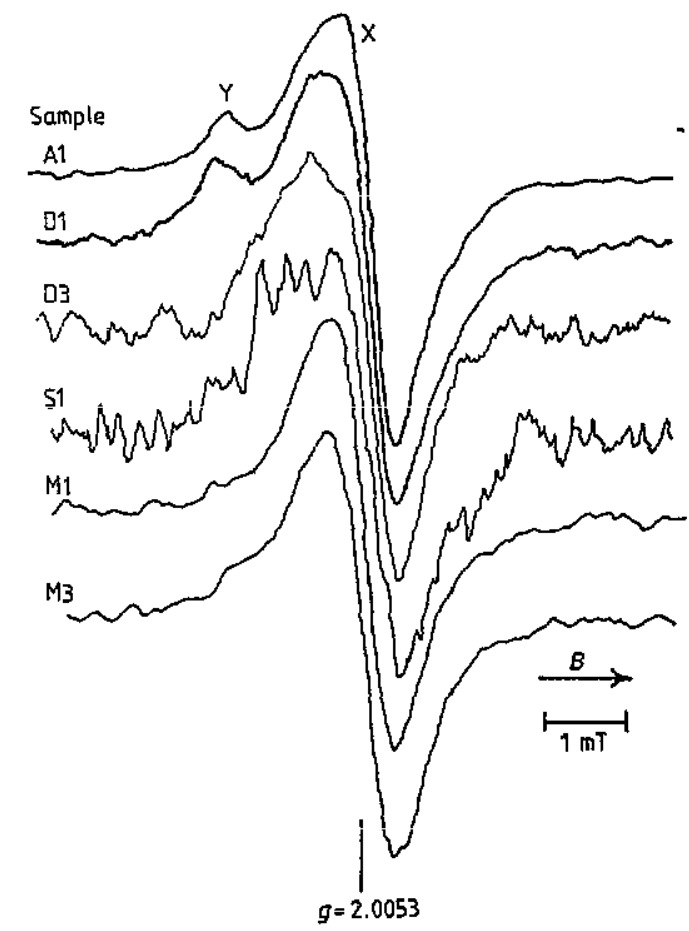

Figure 2. Resonant change in photocurrent in samples $A 1$, $\mathrm{D} 1, \mathrm{D} 3, \mathrm{~S} 1, \mathrm{M} 1$ and $\mathrm{M} 3 . \mathrm{v} \simeq 9.6 \mathrm{GHz}, \mathrm{B} \|[011]$.

value of $2.0053 \pm 0.0003$ and $\mathrm{Y}$ has $\Delta B_{\mathrm{pp}}=0.51 \mathrm{mT}$ and $g=2.014$ (corresponding to the mid-point of the line). As $B$ is rotated in the (011) plane from $B \|[011]$ to $\|[100]$ the feature $Y$ moves to higher fields and at $B \|[100]$ has $g=2.011$. Apart from a slight shift in $g$ value to $2.0056 \pm 0.0003$ for $B \|[100]$ there is little change in the dominant feature $X$. The current always increased at resonance.

It is argued later in section 5.2 that the resonant change in the (dark) conductivity is due to spin-dependent generation (SDG) in a space charge region at one of the contacts. However, as it is not known whether such a region covers all or only part of the area beneath the contact pad it was not felt to be worthwhile to make detailed measurements on this signal. It is worth noting, however, that for some samples the fractional change in conductivity $(\Delta \sigma / \sigma)$ for the main feature $\mathrm{X}$ reached $\sim 10^{-4}$.

\subsection{Resonant change in the photoconductivity}

4.2.1. Resonance at $B \simeq 0.34 \mathrm{~T}$. Figure 2 shows the signals corresponding to a resonant change in photocurrent, $I_{\mathrm{p}}$, at constant voltage, for samples from the CVD-grown wafers A1, D1, D3 and S1 and from two of the MBEgrown wafers $\mathrm{M} 1$ and $\mathrm{M} 3$. All these spectra were recorded at room temperature with $B \|[011], 1 \mathrm{kHz}$ field modulation and a microwave frequency of about $9.6 \mathrm{GHz}$. The spectra for the samples D3, S1, M1 and M3 are similar and consist of a single line with $\Delta B_{\mathrm{p} \bar{p}}=(0.8 \pm 0.1) \mathrm{mT}$ for $\mathrm{S} 1, \mathrm{D} 3$ and $\Delta B_{\mathrm{pp}}=(0.72 \Omega 0.06) \mathrm{mT}$ for $\mathrm{Ml}, \mathrm{M} 3$ and with zero crossing $g$ value of $2.0053 \pm 0.0003$ for $B \|[011]$. The line is asymmetric, being broader on the low-field side and the asymmetry is more marked for the CVD samples $\mathrm{S1}, \mathrm{D} 3$ than for the MBE ones M1, M3. The spectrum for M2 is 


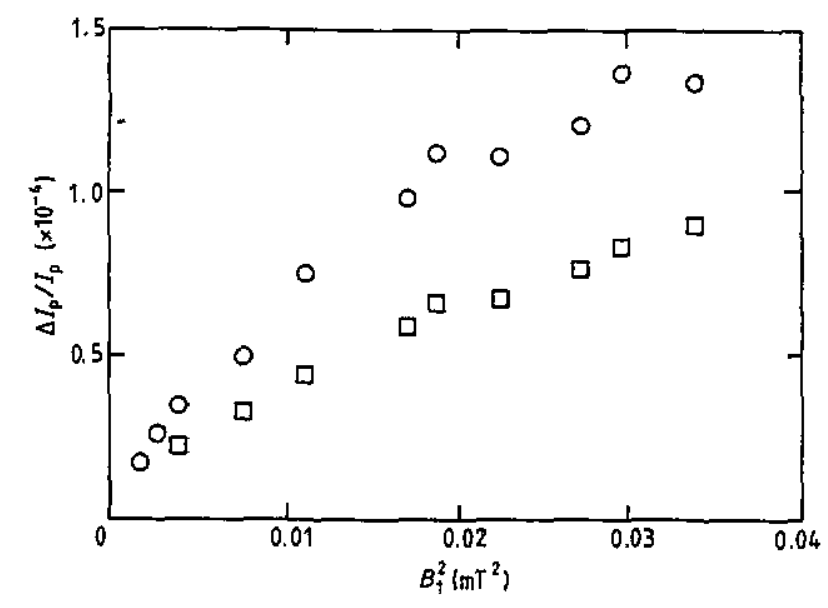

Figure 3. Dependence of $\Delta /_{p} / I_{p}$ (for line $X$ ) on microwave power for the MBE-grown samples $M 1(\square)$ and $M 3(O) . B_{1}$ is the amplitude of the linearly polarized microwave field.

identical to that for $\mathrm{M} 1$ and $\mathrm{M} 3$. Rotation of the magnetic field in the (0i1) plane from $B \|[011]$ to $\|[100]$ causes the $g$ value to increase slightly to $2.0062 \pm 0.0004$ and the line becomes more nearly symmetric but otherwise there is little change. Figure 2 shows that samples Al and D1 have spectra of almost identical shape; the spectrum of the other high-resistivity CVD wafer, D2, is the same as that of D1. The feature $\mathrm{X}$ is the same as that measured in the dark for $A 1, D 1$ and D2 and figure 2 shows that it is very similar to the single line seen in the other samples. Feature $Y$ is also seen in the spectra of these three types of sample but a comparison to the spectrum in figure 1 shows that it is less intense relative to $\mathrm{X}$ than it is when recorded in the dark.

Most measurements were made for a light intensity in the range $50-80 \mathrm{~mW} \mathrm{~cm}^{-2}$ and, as mentioned earlier, the photocurrent is then comparable to the dark current in all the MBE-grown samples and considerably exceeds it in the low-conductivity, CVD-grown samples A1, DI and D2 but for the high-conductivity CVD samples $\mathrm{A} 2, \mathrm{S1}, \mathrm{S} 2$ and D3 the photocurrent did not exceed about $1 \%$ of the dark current. As the noise tended to increase as the total current increased the signal-to-noise ratio was lower for these latter samples than for the other samples. Most measurements were therefore made on all or some of the MBE samples and on the low-conductivity CVD samples rather than on $\mathrm{A} 2, \mathrm{~S} 1, \mathrm{~S} 2$ and $\mathrm{D} 3$.

In every case the photocurrent was found to decrease at resonance. The photocurrent, $I_{\mathrm{p}}$, and the change, $\Delta I_{\mathrm{p}}$, at resonance varied linearly with the applied voltage, $V$, for both polarities of $V$ and hence $\Delta I_{\mathrm{p}} / I_{\mathrm{p}}$ was found to be

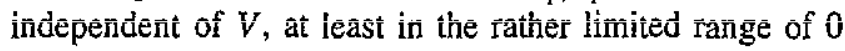
to $\pm 6 \mathrm{~V}$ which was used. Here and in what follows, $\Delta I_{\mathrm{p}}$ refers to the main feature $X$.

Figure 3 shows the dependence of the ratio $\Delta I_{p} / I_{p}$ on the amplitude, $B_{1}$, of the linearly polarized microwave magnetic field at the samples $M 1$ and $M 3$. The spectra were recorded using $1 \mathrm{kHz}$ field modulation and $\Delta I_{\mathrm{p}}$ was determined from the integral over field of each spectrum. The values of $B_{1}$ were calculated following the procedure, given in the book by Poole [14], which relates $B_{1}$ to the $Q$ of the loaded cavity $(\simeq 3 \hat{000})$ and the power incident on

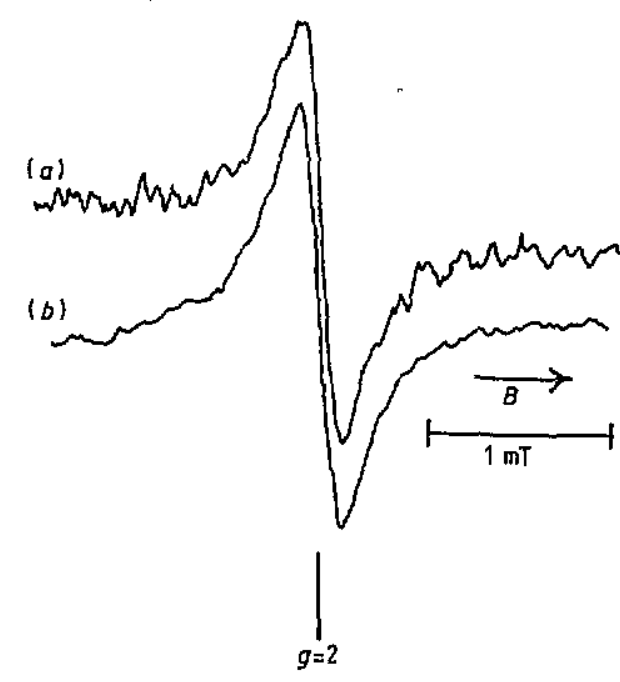

Figure 4. Resonant change in photocurrent in sample $M 1$ (a) and D1 (b). $v \simeq 92 \mathrm{MHz}$.

the cavity ( $\simeq 0.7 \mathrm{~W}$, at $0 \mathrm{~dB}$ attenuation in our case). The $B_{1}$ values are, however, based on the assumption, which may not be valid, that the field configuration is exactly that for the $\mathrm{TE}_{011}$ mode. The dependence shown in figurc 3 is typical of that measured for the other samples $M 2, A 1$, $\mathrm{D} 1$ and D2. Since a limiting, saturation value, for $\Delta I_{\mathrm{p}} / I_{\mathrm{p}}$ has not been reached all we can give are the maximum values obtained, at $B \simeq 0.34 \mathrm{~T}$, for this ratio. These values are $(0.89 \pm 0.18) \times 10^{-4},(0.95 \pm 0.16) \times 10^{-4}$ and (1.4 \pm 0.1$) \times 10^{-4}$ for the three MBE samples M1, M2 and M3 respectively and $(2.0 \pm 0.4) \times 10^{-4},(0.5 \pm 0.3) \times 10^{-4}$, $(0.4 \pm 0.3) \times 10^{-4},(0.5 \pm 0.3) \times 10^{-4},(1.7 \pm 0.5) \times 10^{-4}$, $(1.5 \pm 0.5) \times 10^{-4}$ and $(1.2 \pm 0.5) \times 10^{-4}$ for the CVD samples A1, A2, S1, S2, D1, D2 and D3 respectively. Since the applied voltages are constant these changes are also the fractional changes in photoconductivity $\left(\Delta \sigma_{\mathrm{p}} / \sigma_{\mathrm{p}}\right)$. There was little increase in the linewidth with increasing microwave power and, for example, for each of the three samples M1, M2, M3, the linewidth was only found to increase from $(0.72 \pm 0.06) \mathrm{mT}$ at low power up to $(0.80 \pm 0.06) \mathrm{mT}$ at the highest power.

For A1, D1, D2 and D3 $\Delta I_{\mathrm{p}} / I_{\mathrm{p}}$ increased by about a factor of 5 as the temperature was decreased from 300 to $200 \mathrm{~K}$. These samples also showed a weak dependence of $\Delta I_{\mathrm{p}} / I_{\mathrm{p}}$ on the light intensity; as the intensity was increased from 20 to $80 \mathrm{~mW} \mathrm{~cm}{ }^{-2} \Delta I_{\mathrm{p}} / I_{\mathrm{p}}$ decreased by a factor of about 1.4 but for the MBE samples no change was observed in this intensity range.

4.2.2. Resonance at $B \simeq 3.3 \mathrm{mT}$. Figure 4 shows the resonant change in photocurrent which is centred, as expected for $g \simeq 2$, at a field of about $3.3 \mathrm{mT}$ as a result of replacing the microwave cavity with a coil producing a radiofrequency (RF) field at about $92 \mathrm{MHz}$. The figure shows the resonances obtained for samples $M 1$ and D1 and the width, shape and position of the resonances for samples M2, M3, A1 and D2 are almost identical. For all these samples the linewidth at low RF power is significantly narrower than it is at $B \simeq 0.34 \mathrm{~T}$ and the shape is very close to Lorentzian. The spectra in figure 4 were recorded 
Table 2. Values at room temperature of the parameter $\frac{1}{4} \gamma_{1}^{2} T^{*} \tilde{T}$ obtained from fits to the power dependence of $\delta I_{p}$ and $\Delta B_{p p}$ and values of $\Delta B_{\mathrm{pp}}^{0}$ obtained from the fit and also by direct measurement.

\begin{tabular}{|c|c|c|c|c|}
\hline \multirow[b]{2}{*}{ Wafer } & \multicolumn{2}{|c|}{$\frac{1}{4} \gamma_{1}^{2} T^{*} \tilde{T}(\mathrm{mT})^{-2}$} & \multicolumn{2}{|c|}{$\Delta B_{\mathrm{pp}}^{0}(\mathrm{mT})$} \\
\hline & $\delta I_{\mathrm{p}}$ fit & $\Delta B_{\mathrm{pp}}$ fit & $\Delta B_{\mathrm{pp}}$ fit & $\begin{array}{l}\text { Direct } \\
\text { measurement }\end{array}$ \\
\hline $\begin{array}{l}\text { M1 } \\
\text { M2 } \\
\text { M3 } \\
\text { A1 } \\
\text { D1 }\end{array}$ & $\begin{array}{l}60 \pm 10 \\
70 \pm 10 \\
70 \pm 10 \\
70 \pm 10 \\
50 \pm 10\end{array}$ & $\begin{array}{r}60 \pm 10 \\
70 \pm 10 \\
70 \pm 10 \\
110 \pm 15 \\
75 \pm 10\end{array}$ & $\begin{array}{l}0.14 \\
0.14 \\
0.14 \\
0.12 \\
0.13\end{array}$ & $\begin{array}{l}0.12 \pm 0.02 \\
0.12 \pm 0.02 \\
0.12 \pm 0.02 \\
0.12 \pm 0.02 \\
0.13 \pm 0.02\end{array}$ \\
\hline
\end{tabular}

at room temperature with field modulation at $1 \mathrm{kHz}$ and a light intensity of $80 \mathrm{~mW} \mathrm{~cm}^{-2}$. No orientation dependence of the spectra was detected and the ratio $\Delta I_{\mathrm{p}} / I_{\mathrm{p}}$ was again independent of the voltage, $V$, in the range 0 to $\pm 6 \mathrm{~V}$. Figure $5(a)$ shows how, at constant $I_{\mathrm{p}}$, the peak-to-peak height, $\delta I_{\mathrm{p}}$, of the signal for sample M3 varies with the amplitude, $B_{1}$, of the linearly polarized RF field. The values of $\delta I_{\mathrm{p}}$ are scaled to make the maximum value equal to 1. Figure $5(b)$ shows how the peak-to-peak linewidth of the signal for M3 varies with $B_{1}$. Almost identical results were obtained for the other samples $\mathrm{M} 1, \mathrm{M} 2, \mathrm{Al}$ and $\mathrm{DI}$. The maximum values of $\Delta I_{\mathrm{p}} / I_{\mathrm{p}}$ are $(3.3 \pm 0.6) \times 10^{-4}$, $(2.7 \pm 0.5) \times 10^{-4}$ and $(3.7 \pm 0.6) \times 10^{-4}$ for the three MBE wafers $\mathrm{M} 1, \mathrm{M} 2$ and $\mathrm{M} 3$ respectively and $(2.1 \pm 0.5) \times 10^{-4}$, $(1.7 \pm 0.9) \times 10^{-4},(2.0 \pm 0.5) \times 10^{-4},(1.4 \pm 0.3) \times 10^{-4}$ and $(1.4 \pm 0.7) \times 10^{-4}$ for the CVD wafers A1, S1, D1, $\mathrm{D} 2$ and D3 respectively. The error bars include the slight spread in the values obtained for different samples taken from the same wafer. Figures $5(a)$ and $5(b)$ show that the power dependence of $\delta I_{\mathrm{p}}$ and $\Delta B_{\mathrm{pp}}$ can be fitted quite well to the expressions

$$
\delta I_{\mathrm{p}} \propto \frac{1}{4} \gamma_{1}^{2} B_{1}^{2} T^{*} \tilde{T}\left(1+\frac{1}{4} \gamma_{1}^{2} B_{1}^{2} T^{*} \tilde{T}\right)^{-3 / 2}
$$

and

$$
\Delta B_{\mathrm{pp}}=\Delta B_{\mathrm{pp}}^{0}\left(1+\frac{1}{4} \gamma_{1}^{2} B_{1}^{2} T^{*} \tilde{T}\right)^{1 / 2} .
$$

$\gamma$ is the gyromagnetic ratio $\left(=g \mu_{\mathrm{B}} / h\right)$ and $\mu_{\mathrm{B}}$ is the Bohr magneton. The justification for using these expressions and the processes which contribute to the relaxation times $T^{*}$ and $\tilde{T}$ are discussed in section 5.1. The two parameters which can be adjusted to get the best fit to the linewidth variation are $\Delta B_{\mathrm{pp}}^{0}$ and $\frac{1}{4} \gamma_{1}^{2} T^{*} \tilde{T}$. Apart from a constant scaling factor $\frac{1}{4} \gamma_{1}^{2} T^{*} \tilde{T}$ is the only parameter which can be varied to get the best fit to the power dependence of $\delta I_{\mathrm{p}}$. Table 2 gives the values of these parameters obtained from fits to the data for M1, M2, M3, A1 and D1, and it can be seen that there is no significant difference between the values for these samples. Only for M2 was the power dependence of $\delta I_{\mathrm{p}}$ and $\Delta B_{\mathrm{pp}}$ measured at low temperature $(118 \mathrm{~K})$ as well as at room temperature $(300 \mathrm{~K})$, but no change in minimum linewidth or saturation behaviour could be detected.
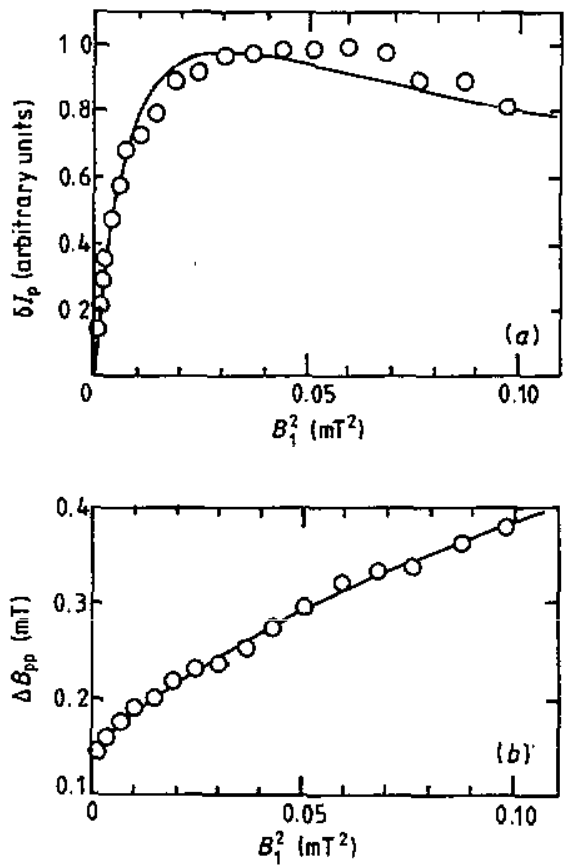

Figure 5. The dependence on RF power of (a) the peak-to-peak signal height, $\delta I_{p}$, normalized to a maximum value of one and $(b)$ the peak-to-peak linewidth $\Delta B_{p p} . B_{1}$ is the amplitude of the linearly polarized RF field. The results are for sample $M 3 . v=92 \mathrm{M} H \mathrm{iz}$. The fits are obtained using expressions given in the text.

\section{Mechanisms for resonance}

\subsection{Rēsonantêt change in photoconductivity}

The most important features of the resonant change in photoconductivity are that it corresponds to a decrease in photoconductivity, that the maximum fractional change in the photoconductivity is of the order of $10^{-4}$ and that this ratio is hardly affected by changing the resonance field by a factor of 100 . These features can be explained by the model proposed by Kaplan, Solomon and Mott (KSM) [2] which accounts for all these features. The heart of their model is the electron-hole pair state in which the electron and hole are close together. It is envisaged that the injected excess carriers form such pair states before the pairs recombine or dissociate and that the electron and hole in such a pair recombine only with each other and not with other holes or electrons. Since in silicon the spin-orbit interaction is small, the total spin, $S$, is conserved during recombination so that if after recombination $S=0$ the recombination will occur if the initial pair state is a singlet with $S=0$ but not if it is a triplet state with $S=1$. This will cause the triplet/singlet population ratio to exceed its isotropic value of $3 / 1$. Saturation of either the electron or hole resonance will restore this isotropic ratio and hence lead to an increase in the recombination rate. L'vov et al [4] have given a quantum mechanical treatment of the KSM model in which they also include the effect of spin relaxation. They exclude any spin-spin interaction, which is justified in the present case as no sign of such interaction is detected. Two characteristic times, $T^{*}, \tilde{T}$, appear in their treatment and are defined by

$$
1 / T^{*}=1 / \tilde{T}+\frac{1}{4} W_{\mathrm{s}} \quad 1 / \tilde{T}=1 / T_{\mathrm{s}}+W_{\mathrm{D}}
$$


where $W_{s}$ is the recombination rate of a pair in a pure singlet state, $W_{\mathrm{D}}$ is the spin-independent dissociation rate of a pair and $T_{\mathrm{s}}$ is the spin relaxation time. They show that for $\omega_{12} \tilde{T} \gg 1$ (where $\omega_{12}=\omega_{1}-\omega_{2}, \omega_{1}=\gamma_{1} B, \omega_{2}=\gamma_{2} B$ and $\gamma_{1}, \gamma_{2}$ are the gyromagnetic ratios for electron and hole) and for the microwave or RF frequency $\omega$ close to $\omega_{1}$ so that $\left(\omega_{2}-\omega\right) \bar{T} \gg 1$ the recombination rate, $R$, via the pair states is given by

$$
R=G\left(2+\frac{4 W_{D}}{W_{\mathrm{s}}}-\frac{1}{1+W_{\mathrm{D}} T_{\mathrm{s}}}-W_{\mathrm{D}} \tilde{T} f\left(B_{1}^{2}\right)\right)^{-1}=G \Phi
$$

where

$$
f\left(B_{1}^{2}\right)=\frac{1}{4} \gamma_{1}^{2} T^{*} \tilde{T} B_{1}^{2}\left\{1+\left[\left(\omega-\omega_{1}\right) T^{*}\right]^{2}+\frac{1}{4} \gamma_{1}^{2} T^{*} \tilde{T} B_{1}^{2}\right\}^{-1}
$$

and $G$ is the generation rate of the pairs. The expression for $R$ in the vicinity of the other resonance has the same form, with $\gamma_{1}, \omega_{1}$ replaced by $\gamma_{2}, \omega_{2}$ respectively.

Kaplan et al [2] suppose that the generation rate of the pairs depends only on the concentrations of the excess, injected, carriers. If we suppose that the excess free electron and hole concentrations are both equal to $\Delta n$ and that $G \propto \Delta n$ then, as the photoconductivity $\sigma_{\mathrm{p}} \propto \Delta n$, we have that $R \propto \sigma_{\mathrm{p}} \Phi$. Since the light intensity is kept constant then so too is the generation rate of free electronhole pairs, and as steady-state conditions are maintained then $R$ is also constant. Since $R \propto \sigma_{\mathrm{p}} \Phi$ then $\Delta \sigma_{\mathrm{p}}(\omega) / \sigma_{\mathrm{p}}=$ $-\Delta \Phi / \Phi$. If there are additional spin-independent or weakly dependent pathways then the fractional change in $\sigma_{\mathrm{p}}$ will be less than the fractional change in $\Phi$. In practice $\Delta \sigma_{\mathrm{p}} / \sigma_{\mathrm{p}} \ll 1$ and if also $\Delta \Phi / \Phi \ll 1$ then equations (4) and (5) imply that

$$
\begin{gathered}
\frac{\Delta \sigma_{\mathrm{p}}(\omega)}{\sigma_{\mathrm{p}}} \simeq-W_{\mathrm{D}} \tilde{T}\left(2+\frac{4 W_{\mathrm{D}}}{W_{\mathrm{s}}}-\frac{1}{1+W_{\mathrm{D}} T_{\mathrm{s}}}\right)^{-1} \\
\times \frac{\frac{1}{4} \gamma_{1}^{2} B_{1}^{2} T^{*} \tilde{T}}{1+\left[\left(\omega_{1}-\omega\right) T^{*}\right]^{2}+\frac{1}{4} \gamma_{1}^{2} B_{1}^{2} T^{*} \tilde{T}} .
\end{gathered}
$$

The exact expression for $\Delta \sigma_{\mathrm{p}} / \sigma_{\mathrm{p}}$ at the centre of the resonance at saturation is

$$
\left(\frac{\Delta \sigma_{\mathrm{p}}}{\sigma_{\mathrm{p}}}\right)_{\mathrm{sat}}=-\frac{W_{\mathrm{s}}}{W_{\mathrm{s}}+4 W_{\mathrm{D}}} \frac{W_{\mathrm{D}} T_{\mathrm{s}}}{1+W_{\mathrm{D}} T_{\mathrm{s}}} .
$$

Equation (6) predicts that $\Delta \sigma_{\mathrm{p}}(\omega)$ is negative, that its peak value, $\Delta \sigma_{\hat{p}}$, is field independent and that $\Delta \sigma_{\bar{p}} / \sigma_{\hat{p}}$ is independent of the voltage $V$, all of which are consistent with the results given earlier.

Since the use of field modulation gives a signal proportional to $\mathrm{d} \sigma_{\mathrm{p}} / \mathrm{d} B$ it is convenient to take the derivative of equation ( 7 ) and hence obtain

$$
\frac{\mathrm{d}\left(\Delta \sigma_{\mathrm{p}}\right)}{\mathrm{d} B} \propto \frac{\frac{1}{4} \gamma_{1}^{2} B_{1}^{2} T^{*} \tilde{T}\left(\omega-\omega_{1}\right) T^{*}}{\left\{1+\left[\left(\omega_{1}-\omega\right) T^{*}\right]^{2}+\frac{1}{4} \gamma_{1}^{2} B_{1}^{2} T^{*} \tilde{T}\right\}^{2}}
$$

Equation (8) yields a resonance line with peak-topeak height, $\delta I_{\mathrm{p}}$, and peak-to-peak width, $\Delta B_{\mathrm{pp}}$, given by equations (1) and (2) respectively with $\Delta B_{\mathrm{pp}}^{0}=$ $2 /\left(\sqrt{3} \gamma_{1} T^{*}\right)$.

It is reasonable that this, homogeneous, linewidth should depend on $W_{s}$ and $W_{D}$ since they, as well as $T_{s}$, affect the lifetime of the pair state. The fitting procedure used earlier for $\delta I_{\mathrm{p}}$ and $\Delta B_{\mathrm{pp}}$ is only valid if the RF line is homogeneously broadened and we now consider whether this assumption is justified. The fact that the microwave resonance line $X$ is appreciably broader than the RF line at low RF power suggests that there is a contribution to its width arising from a spread, $\Delta g$, in the $g$ value since such a spread will give rise to a spread in the line positions which is proportional to the field, and hence the frequency, at resonance. If we assume that the $g$-value spread gives rise to a Gaussian spread of Lorentzian lines then the overall line shape is given by the convolution of the Lorentzian line with a Gaussian function. This produces the so-called Voight line and Stoneham [15] has shown that to a good approximation its total linewidth is given by

$$
\Delta B_{\mathrm{pp}}^{\mathrm{Tot}}=\frac{\left(\Delta B_{\mathrm{pp}}^{\mathrm{G}}\right)^{2}+0.9085 \Delta B_{\mathrm{pp}}^{\mathrm{G}} \Delta B_{\mathrm{pp}}^{\mathrm{L}}+0.4621\left(\Delta B_{\mathrm{pp}}^{\mathrm{L}}\right)^{2}}{\Delta B_{\mathrm{pp}}^{\mathrm{G}}+0.4621 \Delta B_{\mathrm{pp}}^{\mathrm{L}}} .
$$

Taking $\Delta B_{\mathrm{pp}}^{\mathrm{Tot}}=0.70 \mathrm{mT}$ at $9.6 \mathrm{GHz}$ and $0.13 \mathrm{mT}$ at $92 \mathrm{MHz}$ and taking $\Delta B_{\mathrm{pp}}^{\mathrm{G}}=E v$, where $E$ is a constant and $\nu$ is the RF or microwave frequency, gives $E=6.6 \times 10^{-11} \mathrm{mT} \mathrm{Hz}{ }^{-1}$ and $\Delta B_{\mathrm{pp}}^{\mathrm{L}}=0.13 \mathrm{mT}$. This gives $\Delta B_{\mathrm{pp}}^{\mathrm{G}}=0.63 \mathrm{mT}$ at $9.6 \mathrm{GHz}$ corresponding to a spread, $\Delta g$, in $g$ of about 0.004 but at $92 \mathrm{MHz} \Delta B_{\mathrm{pp}}^{\mathrm{G}}$ is only $0.006 \mathrm{mT}$ and it is therefore a good approximation to neglect it. Nevertheless, small contributions to the linewidth from inhomogeneous broadening, caused, for example, by unresolved ${ }^{29} \mathrm{Si}$ hyperfine lines, may remain and therefore we take the linewidth $\Delta B_{\mathrm{pp}}^{0}$ to give a lower limit to $T^{*}$. Since there does not appear to be any significant difference between the values, given in table 2 , of $\Delta B_{\mathrm{pp}}^{0}$ and $\frac{1}{4} \gamma_{1}^{2} T^{*} \tilde{T}$ for the wafers $\mathrm{M} 1, \mathrm{M} 2, \mathrm{M} 3, \mathrm{Al}$ and D1 we take the average values and obtain

$$
\frac{2}{\sqrt{3} \gamma_{1} T^{*}} \leq 0.13 \pm 0.03 \mathrm{mT}
$$

and

$$
\frac{1}{4} \gamma_{1}^{2} T^{*} \tilde{T}=70 \pm 20(\mathrm{mT})^{-2}
$$

Taking $\gamma_{1}=1.764 \times 10^{11} \mathrm{~s}^{-1} \mathrm{~T}^{-1}$ corresponding to $\mathrm{g}=$ 2.0055 gives $T^{*} \gtrsim 5 \times 10^{-8} \mathrm{~s}, T^{*} \tilde{T}=(9 \pm 3) \times 10^{-15} \mathrm{~s}^{2}$ and hence $\tilde{T} \lesssim 2 \times 10^{-7} \mathrm{~s}$; also equation (3) implies that $\tilde{T} \gtrsim T^{*}$ and therefore $5 \times 10^{-8} \mathrm{~s} \leq \tilde{T} \leq 2 \times 10^{-7} \mathrm{~s}$. Since $W_{\mathrm{s}}=4\left[\left(T^{*}\right)^{-1}-(\tilde{T})^{-1}\right]$ then $0 \leq W_{\mathrm{s}} \leq 6 \times 10^{7} \mathrm{~s}^{-1}$. Furthermore $W_{\mathrm{D}}=(\tilde{T})^{-1}-T_{s}^{-1}$. If $W_{\mathrm{D}}$ has the form corresponding to the emission to the conduction band of an electron from a level at energy $\triangle E$ below the band edge [16] then

$$
W_{\mathrm{D}}=\sigma_{n} V_{\mathrm{tb}} N_{\mathrm{c}} \exp (-\Delta E / k T)
$$

where the symbols have their usual meanings. At $T=$ $300 \mathrm{~K}, V_{t \mathrm{~h}}=2 \times 10^{5} \mathrm{~m} \mathrm{~s}^{-1}$, so with $N_{\mathrm{c}}=2.8 \times 10^{25} \mathrm{~m}^{-3}$ and taking $\sigma_{n}=10^{-20} \mathrm{~m}^{2}$, a typical value, equation (10) implies that if, for example, $W_{\mathrm{D}}=10^{7} \mathrm{~s}^{-1}$ then $\Delta E=0.22 \mathrm{eV}$. With $\Delta E=0.22 \mathrm{eV}$ equation (10) implies that $W_{D}$ should drop by a factor of about $5 \times 10^{5}$ as $T$ falls from $300 \mathrm{~K}$ to $118 \mathrm{~K}$. However, as mentioned in section 4.2 .2 , there was no discernible change in the minimum linewidth or saturation behaviour of the RF line for wafer 
M2 when $T$ was reduced to $118 \mathrm{~K}$. Therefore we must have $W_{\mathrm{D}} \ll \tilde{T}^{-1}$ and so $T_{\mathrm{s}} \simeq \tilde{T} \sim 10^{-7} \mathrm{~s}$ and $W_{\mathrm{D}} \ll 10^{7} \mathrm{~s}^{-1}$. Further information can be obtained using equation (7) for the saturation value of $\Delta \sigma_{\mathrm{p}} / \sigma_{\mathrm{p}}$. Since we have shown above that $W_{\mathrm{D}} T_{\mathrm{s}} \ll i$ this becomes

$$
\left(\frac{\Delta \sigma_{\mathrm{p}}}{\sigma_{\mathrm{p}}}\right)_{\text {sat }}=-\frac{W_{\mathrm{s}} W_{\mathrm{D}} T_{\mathrm{s}}}{W_{\mathrm{s}}+4 W_{\mathrm{D}}}
$$

If $W_{\mathrm{s}} \gg 4 W_{\mathrm{D}}$ then $\left(\Delta \sigma_{\mathrm{p}} / \sigma_{\mathrm{p}}\right)_{\text {sat }} \simeq-W_{\mathrm{D}} T_{\mathrm{s}}$ and it would be expected that $\Delta \bar{\sigma}_{\mathrm{p}} / \tilde{\sigma}_{\mathrm{p}}$ would decrease rapidily as the temperature is decreased whereas in fact $\Delta \sigma_{p} / \sigma_{p}$ increases. However, if the other inequality holds, $W_{s} \leq 4 W_{D}$, then $\Delta \sigma_{\mathrm{p}} / \sigma_{\mathrm{p}} \simeq-\frac{1}{4} W_{\mathrm{s}} T_{\mathrm{s}} ;\left|\Delta \sigma_{\mathrm{p}} / \sigma_{\mathrm{p}}\right|$ would then be expected to increase as $T$ decreased since $T_{s}$ should increase whereas $W_{\mathrm{s}}$ might not change much. Taking $\left|\Delta \sigma_{\mathrm{p}} / \sigma_{\mathrm{p}}\right| \simeq 2 \times 10^{-4}$ and $T_{s} \simeq \tilde{T}$ gives $4 \times 10^{3} \mathrm{~s}^{-1} \leq W_{s} \leq 1.6 \times 10^{4} \mathrm{~s}^{-1}$. Having $T_{s}^{-1} \gg W_{s}$ considerably reduces $\left|\Delta \sigma_{\mathrm{p}} / \sigma_{\mathrm{p}}\right|$ below its theoretical maximum value but with $\frac{1}{4} W_{s} T_{s} \simeq 2 \times$ $10^{-4}$ it is still much larger than $\sim 10^{-6}$ expected if the system was fully thermalized (at $300 \mathrm{~K}$ ) which would make the $\left|\Delta \sigma_{p} / \sigma_{p}\right|$ strongly field dependent. Of course, if recombination via the paired state is not the dominant mechanism then $|\Delta \Phi / \Phi|>\left|\Delta \sigma_{p} / \sigma_{p}\right|$ and the upper limit for $W_{\mathrm{s}}$ would be larger. The final values deduced for $T^{*}$, $\tilde{T}, T_{\mathrm{s}}, W_{\mathrm{D}}$ and $W_{\mathrm{s}}$ may be summarized as

$$
\begin{aligned}
& T^{*} \gtrsim 5 \times 10^{-8} \mathrm{~s} \quad 2 \times 10^{-7} \mathrm{~s} \gtrsim \tilde{T} \gtrsim 5 \times 10^{-8} \mathrm{~s} \quad T_{\mathrm{s}} \simeq \tilde{T} \\
& W_{\mathrm{D}} \ll 10^{7} \mathrm{~s}^{-1} \text { and } 4 \times 10^{3} \mathrm{~s}^{-1} \leq W_{\mathrm{s}} \ll 4 \times 10^{7} \mathrm{~s}^{-1} \\
& \text { since } W_{\mathrm{s}} \ll 4 W_{\mathrm{D}} .
\end{aligned}
$$

These values refer to the pair state associated with the feature $\mathrm{X}$ since, even when $\mathrm{Y}$ is present, $\mathrm{X}$ is the dominant feature.

We can now check to see if the condition $\omega_{12} \tilde{T} \gg 1$, for which equation (4) is valid, is likely to be valid for the $\mathrm{RF}$ data. Since $\tilde{T} \simeq T^{*}$ then the condition $\omega_{12} \tilde{T} \gg 1$ is equivalent to the requirement that the separation between the 'electron' and 'hole' resonances is greater than their width and they should therefore be resolved. However, as was the case for plastically deformed silicon $[3,4]$, we only detect one RF line. This may be because, even at $B=3.3 \mathrm{mT}$, the hole resonance is too broad to detect and in this case any overlap of the two resonances may indeed be small so that the above condition is valid. However, another possibility is that the two resonances do largely overlap both at $9.6 \mathrm{GHz}$ and $92 \mathrm{MHz}$. In this latter case the condition $\omega_{12} \tilde{T} \gg 1$ is not satisfied and the analysis is not strictly valid. However since the quantities $T_{\mathrm{s}}, W_{\mathrm{s}}$ and $W_{D}$ refer to the pair then both the width and saturation behaviour of the RF line will still be determined only by $T_{s}, W_{s}$ and $W_{D}$ and the values given above will still be approximately correct.

\subsection{Resonant change in conductivity}

The resonant increase, $\Delta \sigma$, in conductivity occurs when one of the contacts behaves like a reverse biased diode. It is therefore likely to arise from SDG in the depletion region which has already been suggested to explain the resonance obvserved in reverse biased pn junction diodes [17]. The fact that we find $\Delta \sigma / \sigma$ reaches $\sim 10^{-4}$ indicates that it may also arise from the KSM model.

\section{Discussion}

We have seen that the KSM model can account for the resonant changes we have observed, but a number of topics-ithe nature of the defects involved, the recombination pathway, a comparison of the various samples and how the results compare with those obtained in other cases-all need to be discussed and we now consider each of these in turn.

\subsection{Nature of defects and SDR or SDG pathways}

The first question to be answered is what corresponds to the 'electron' and 'hole' in each of the intermediate pair states corresponding to features $\mathrm{X}$ and $\mathrm{Y}$. We first consider the major resonance $X$. The zero-crossing $g$ value, $g_{0}$, of between 2.005 and 2.006 is typical of Si dangling bond (DB) centres and there is evidence for their presence in the bulk of the $\mathrm{Si}$ layer and possibly at the $\mathrm{Si} / \mathrm{Al}_{2} \mathrm{O}_{3}$ interface $[18,19]$. Some studies [20,21] of as-grown CVD SOS have found evidence for a slight degree of amorphization but the angular dependence of $g_{0}$ excludes the possibility that $\mathrm{X}$ arises from DBs solely in amorphous regions. The magnitude of $\triangle \sigma_{\mathrm{p}} / \sigma_{\mathrm{p}}$ for MBE SOS samples was found to be the same whether the measurement was made a few days or several weeks after the oxide was etched off, and this strongly suggests that $\mathrm{DBs}$ at the Si/native oxide interface play an insignificant role. The most likely source of DBs which cause $\mathrm{X}$ are those associated with the dislocations present in all the samples. Dislocations, unlike microtwins and stacking faults, are not reduced greatly in number by SPEG or DSPEG treatments [22]. Furthermore, studies of plastically deformed silicon show that dislocations give rise to spin-dependent recombination $[3,4,23]$ and many characteristics of the resonance are similar to those we observe. The question now arises of what is the other partner in the pair? There is evidence [24] that the spin-dependent recombination step in a-Si:H is the tunnelling of an electron from a conduction band tail (CBT) state, in which it had been trapped, to a neutral Si DB; holes trapped in valence band tail (VBT) states may also play a role. The tail states are usually attributed to distorted or weak bonds which are diamagnetic when neutral and EPR signals with $g \simeq 2.004$ and $g \simeq 2.01$ are associated with states $\mathrm{CBT}^{-}$and $\mathrm{VBT}^{+}$respectively [25]. There is evidence for tail states in SOS [26], and therefore we suggest that the spin-dependent recombination step in SOS is either $\mathrm{CBT}^{-}+\mathrm{DB}^{0} \rightarrow \mathrm{CBT}^{0}+\mathrm{DB}^{-}$or $\mathrm{VBT}^{+}+\mathrm{DB}^{0} \rightarrow \mathrm{VBT}^{0}+\mathrm{DB}^{+}$where $\mathrm{DB}$ is the dislocation dangling bond. Since dissociation of the pair corresponds to re-emission of the electron or hole into the relevant band and since we have shown that $W_{\mathrm{D}} \ll 10^{7} \mathrm{~s}^{-1}$ then the tail states involved must be quite deep. There may be pairs for which these states are shallow but the higher value of $W_{D}$ would not only reduce the size of $\Delta \sigma_{\mathrm{p}}$ but increase $\Delta B_{\mathrm{pp}}$ so that their contưtibution to the observed resononance would be insignificant. It is for these reasons that the model of Rong et al [7] in which one of the carriers is in a shallow excited state is unlikely to be correct. They take $W_{D} \simeq 10^{10} \mathrm{~s}^{-1}$, which would give a resonance far wider than is observed in Si pn junction diodes. 
The absence of two resolved lines in SOS means either that one of the lines is too broad to be detected (as is likely to be the case for $\mathrm{VBT}^{+}$) or overlaps too closely with the $\mathrm{DB}^{0}$ line (as may be the case for $\mathrm{CBT}^{-}$).

We have not identified the defects responsible for feature $\mathrm{Y}$, but the fact that it is strongest relative to $\mathrm{X}$ for signals recorded in the dark suggests that the defects are mostly in the region below the rectifying contacts where the depletion layer must lie.

The spectra for samples A1, D1 and D2 recorded in the dark are almost identical to those in the light. The same defects must therefore affect both spin dependent generation as well as recombination; the same conclusion has been reached for SDR and SDG observed in Si pn junction diodes [17]. However, neither of the SDR steps given earlier to explain $X$ are spin dependent in the reverse direction and we therefore have no satisfactory explanation for the SDG signal.

\subsection{Comparison of results for the various samples}

Apart from the occurrence of the weak resonance $\mathrm{Y}$ only in samples A1, D1 and D2 it is remarkable how similar are the results for all the samples. The main resonance $X$ and the relaxation times are very nearly the same in all samples. The only difference is that the values of $\Delta \sigma_{\mathrm{p}} / \sigma_{\mathrm{p}}$ are slightly smaller in the high-conductivity CVD SOS samples than in the low-conductivity ones. This may be because shifting the Fermi level away from mid-gap reduces the number of $\mathrm{DB}$ centres in the neutral paramagnetic state and hence reduces $\Delta \sigma_{\mathrm{p}}$.

The photoconductivity, at constant light intensity, of the MBE SOS samples decreases by about a factor of 90 as the thickness of the $\mathrm{Si}$ film is reduced from $0.5 \mu \mathrm{m}$ to $0.15 \mu \mathrm{m}$ so that the fact that $\left(\Delta \sigma_{\mathrm{p}} / \sigma_{\mathrm{p}}\right)_{\mathrm{sat}}$ changes by less than a factor of 1.5 implies either that the spin-dependent pathway is indeed the dominant recombination pathway or possibly that its importance relative to other pathways remains unaltered.

\subsection{Comparison of results with those for other samples}

Table 3 shows that the parameters associated with the main feature, $X$, in the resonant change in photoconductivity are very similar not only to those obtained for an SOS sample prepared by liquid-phase epitaxy (LPE) [5] but also to those obtained for samples of plastically deformed silicon. The near certainty that silicon dangling bonds at or near disiocations are involved in the spin-dependent recombination in the latter samples therefore supports the proposal that they are involved in SOS too. As regards the resonant increase in (dark) conductivity, it is interesting to note that Szkielko [27] also observed such a signal in a sample of plastically deformed silicon which had an indium-alloyed $\mathrm{p}-\mathrm{n}$ junction at one end. As was the case for some of our samples $A 1, D 1$ and $D 2$, he observed the conductivity change to reverse sign from positive to negative when the sample was illuminated. He also supposed that the resonance was due to $\overline{S D G}$ from 
dislocations in the dark and SDR at dislocations with the light on.

\section{Conclusions}

A resonant decrease has been observed in the photoconductivity of all the CVD and MBE SOS samples. The dominant feature, $X$, occurs in all samples and a weaker feature $Y$ occurs only in the high-resistivity CVD samples. The main characteristics of $\mathrm{X}$-its sign and its magnitude, $\Delta \sigma_{\mathrm{p}} / \sigma_{\mathrm{p}}$, of up to $\simeq 10^{-4}$ which is approximately field independent $\longrightarrow$ can be explained by the KSM pair model. Using the quantum mechanical version of this model by L'vov et al [4] we obtain, for the pair associated with feature $\mathrm{X}, T_{\mathrm{s}}=(0.5-2) \times 10^{-7} \mathrm{~s}, W_{\mathrm{s}}=4 \times 10^{3}-4 \times 10^{7} \mathrm{~s}^{-1}$ and $\frac{1}{4} W_{\mathrm{s}} \ll W_{\mathrm{D}} \ll 10^{7} \mathrm{~s}^{-1}$. In addition we show that the width of this feature, when recorded at a microwave frequency of about $9.6 \mathrm{GHz}$, arises primarily for a $g$-value spread, $\Delta g$, of about 0.004 . The pair state corresponding to feature $\mathrm{X}$ is suggested to be an unpaired electron at a Si dangling bond associated with a dislocation and an electron or hole trapped at a nearby tail state defect. The saturation value of $\left|\Delta \sigma_{p} / \sigma_{p}\right|$ differs surprisingly little amongst all the SOS samples despite the fact that $\sigma_{p}$ decreases by about a factor of 90 as the thickness of the $\mathrm{Si}$ film is reduced from $0.5 \mu \mathrm{m}$ to $0.15 \mu \mathrm{m}$ in the MBE samples. This suggests that either the spin-dependent pathway is the dominant recombination pathway or else that its importance relative to other pathways is about the same in all samples.

The three types of CVD SOS samples, A1, D1 and $D 2$, which exhibit nonlinear $I-V$ characteristics also give a resonant increase in the (dark) conductivity. This is interpreted as spin-dependent generation in a space charge region at the contacts. The feature $Y$ is stronger relative to $X$ than it is when these samples are illuminated, which suggests that $\mathrm{Y}$ is associated with defects or impurities concentrated in the contact regions.

\section{Acknowledgments}

CO'R and LB gratefully acknowledge the receipt of research studentships from Trinity College, and LB also thanks the Irish Science and Technology Agency for some financial support.

\section{References}

[1] Lepine D J 1972 Phys. Rev. B 6436

[2] Kaplan D, Solomon I and Mott N F 1978 J. Physique 39 L-51

[3] Mima L S, Strikha V I and Tretyak O V 1980 Sov. Phys.-Semicond. 141328

[4] L'yov V S, Mima L S and Tretyak O V 1982 Sov. Phys.-JETP 56897

[5] Borisov F I, Vorob'ev X Y, Strikha V I, Tretyak O V and Shmatov A A, 1985 Sov. Phys.-Semicond. 19535

[6] Vranch R L, Henderson B and Pepper M 1988 Appl. Phys. Lett. 521161

[7] Rong F C, Buchwald W R, Poindexter E H, Warren W L and Keeble D J 1991 Solid State Electron. 34835

[8] Richmond E D, Pellegrino J G, Twigg M E, Qadri S and Duffy M T 1990 Thin Solid Films 192287

[9] Twigg M E, Richmond E D and Pellegrino J G 1989 Appl. Phys. Lett. 541766

[10] Hodge A M, Cullis A G and Chew N G 1985 Mater. Res. Soc. Symp. Proc. 35393

[11] Twigg M E, Richmond E D and Pellegrino J G 1989 Mater. Res. Soc. Symp. Proc. 138409

[12] Richmond E D, Twigg M E, Qadri S, Pellegrino J G and Duffy M T 1990 Appl. Phys. Lett. 562551

[13] Twigg M E, Richmond E D and Pellegrino J G 1990 J. Appl. Phys. 673706

[14] Poole C P 1967 Electron Spin Resonance (New York: Wiley)

[15] Stoneham A M 1972 J. Phys. D: Appl. Phys. 5670

[16] Sze S M 1985 Semiconductor Devices, Physics and Technology (New York: Wiley)

[17] Rong F, Poindexter E H, Harmatz M, Buchwald W R and Gerardi G J 1990 Solid State Commun. 76 1083

[18] Jastrzebski L, Lagowski J, Cullen G W and Pankove J I 1982 Appl. Phys. Lett. 40713

[19] Lagowski J, Jastrzebski $L$ and Cullen G W 1983 J. Electrochem Soc.: Solid-State Sci. Technol. 1301744

[20] Pickering C, Dixon S, Gasson D B, Robbins D J and Hodge A M 1987 J. Crystal Growth 84180

[21] Lagowski J, Jastrzebski L and Cullen G W 1981 J. Electrochem. Soc.: Solid-State Sci. Technol. 1282665

[22] Richmond E D and Knudson A R $1984 \mathrm{~J}$. Vac. Sci. Technol. A 2569

[23] Wosinski T and Figielski T 1975 Phys. Status Solidi b 71 K73

[24] Dersch H, Schweitzer L and Stuke J 1983 Phys. Rev. B 23 4678

[25] Street R A 1991 Hydrogenated Amorphous Silicon (Cambridge: Cambridge University Press)

[26] Goodman A M 1975 IEEE Trans. Electron Devices 2263

[27] Szkielko W 1978 Phys. Status Solidi b 90 K81 